\title{
SCIENTIFIC REPORTS

\section{Frequency-domain CBE imaging for ultrasound localization of the HIFU focal spot: a feasibility study}

Kun Yang ${ }^{1}$, Qiang Li ${ }^{1}$, Hao-Li Liu $\mathbb{1}^{2}$, Chin-Kuo Chen ${ }^{3}{ }^{3}$, Cheng-Wei Huang ${ }^{4}$, Jheng-Ru Chen ${ }^{4}$, Yu-Wei Tsai ${ }^{4}$, Zhuhuang Zhou ${ }^{5 *} \&$ Po-Hsiang Tsui ${ }^{4,6,7 *}$

High-intensity focused ultrasound (HIFU) is a well-accepted tool for noninvasive thermal therapy. To control the quality of HIFU treatment, the focal spot generated in tissues must be localized. Ultrasound imaging can monitor heated regions; in particular, the change in backscattered energy (CBE) allows parametric imaging to visualize thermal information in the tissue. Conventional CBE imaging constructed in the spatial domain may be easily affected by noises when the HIFU focal spot is visualized. This study proposes frequency-domain $C B E$ imaging to improve noise tolerance and image contrast in HIFU focal spot monitoring. Phantom experiments were performed in a temperaturecontrolled environment. HIFU of $2.12 \mathrm{MHz}$ was applied to the phantoms, during which a clinical scanner equipped with a $3-\mathrm{MHz}$ convex array transducer was used to collect raw image data consisting of backscattered signals for B-mode, spatial-, and frequency-domain CBE imaging. Concurrently, temperature changes were measured at the focal spot using a thermocouple for comparison with CBE values by calculating the correlation coefficient $r$. To further analyze CBE image contrast levels, a contrast factor was introduced, and an independent $t$-test was performed to calculate the probability value $p$. Experimental results showed that frequency-domain CBE imaging performed well in thermal distribution visualization, enabling quantitative detection of temperature changes. The CBE value calculated in the frequency domain also correlated strongly with that obtained using the conventional spatial-domain approach $(r=0.97)$. In particular, compared with the image obtained through the conventional method, the contrast of the $\mathrm{CBE}$ image obtained using the method based on frequencydomain analysis increased by 2.5 -fold $(4 \mathrm{~dB} ; p<0.05)$. Frequency-domain computations may constitute a new strategy when ultrasound CBE imaging is used to localize the focal spot in HIFU treatment planning.

High-intensity focused ultrasound (HIFU) is a therapeutic technique for inducing thermal lesions in tissues by focusing acoustic energy to generate a high temperature at the focal point. HIFU has been increasingly explored as a noninvasive thermal treatment for ablating tumors. HIFU treatment has been established to play a key role in shortening postoperative recovery time and reducing the risk of complications ${ }^{1,2}$.

During HIFU treatment, correct sound beam distribution is essential to avoid thermal damage to surrounding normal tissues. However, phase aberrations caused by tissue inhomogeneities typically make the practical heating point shift from the geometric focus ${ }^{3}$. Therefore, targeting the focal spot is a critical step in HIFU treatment planning. Currently, magnetic resonance (MR) imaging and ultrasound imaging are two major modalities for the localization of the HIFU focal spot in treatment planning ${ }^{4}$. MR imaging is a reliable method for localizing the HIFU focal spot because it allows detection of the focal spot with a temperature change of $1{ }^{\circ} \mathrm{C}^{5,6}$. However, MR imaging is expensive with limited availability, and its device design and operating environment must meet

${ }^{1}$ School of Microelectronics, Tianjin University, Tianjin, China. ${ }^{2}$ Department of Electrical Engineering, Chang-Gung University, Taoyuan, Taiwan. ${ }^{3}$ Department of Otolaryngology - Head and Neck Surgery, Chang Gung Memorial Hospital and Chang Gung University, Taoyuan, Taiwan. ${ }^{4}$ Department of Medical Imaging and Radiological Sciences, College of Medicine, Chang Gung University, Taoyuan, Taiwan. ${ }^{5}$ College of Life Science and Bioengineering, Beijing University of Technology, Beijing, China. ${ }^{6}$ Medical Imaging Research Center, Institute for Radiological Research, Chang Gung University and Chang Gung Memorial Hospital at Linkou, Taoyuan, Taiwan. ${ }^{7}$ Department of Medical Imaging and Intervention, Chang Gung Memorial Hospital at Linkou, Taoyuan, Taiwan. *email: zhouzh@bjut.edu. cn; tsuiph@mail.cgu.edu.tw 


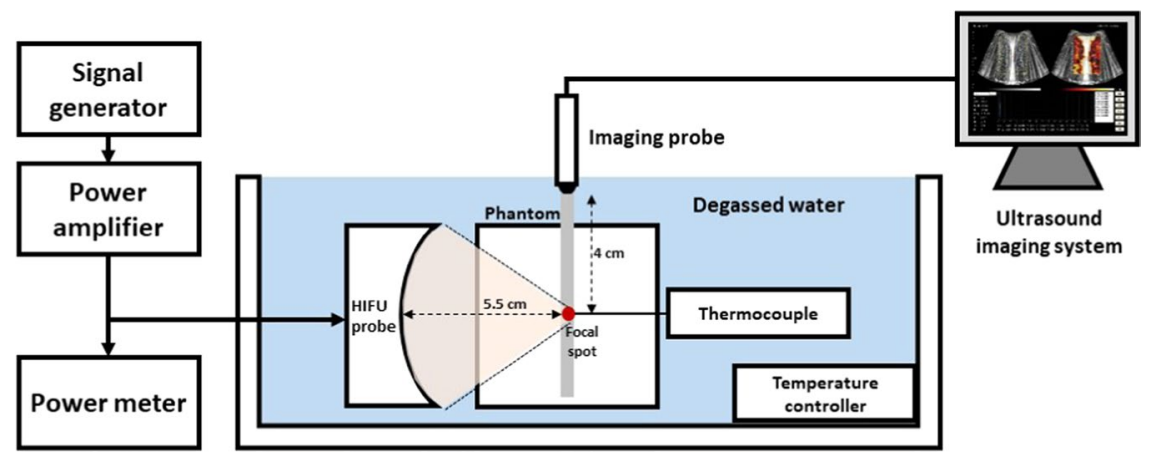

Figure 1. Experimental setup involved a self-assembled HIFU system, a clinical ultrasound system, and a thermocouple. The HIFU system comprised a function generator, a gain-variable power amplifier, a power meter, and a HIFU probe. The HIFU and imaging focal spots were at the same location.

nonferromagnetic requirements for materials and components. By contrast, ultrasound imaging has several advantages in terms of cost-effectiveness, real-time capability, and low compatibility requirements. In particular, the temperature increase alters the properties of ultrasound backscattered signals, including the echo time shift (caused by changes in the tissue thermal expansion and speed of sound $)^{7-9}$, acoustic attenuation ${ }^{10}$, and change in backscattered energy $(\mathrm{CBE})^{11-13}$. This implies that ultrasound parameters may serve as an alternative for localizing the HIFU-induced focal spot.

However, not every ultrasound parameter can be effectively used to monitor the focal spot for HIFU treatment planning. During the planning stage, a small temperature increment is typically generated at the focal spot to avoid heat deposition in the target ${ }^{14}$. The acoustic attenuation coefficient is unsuitable for monitoring the focal spot because it exhibits significant changes only at temperatures exceeding $50^{\circ} \mathrm{C}^{10}$. The echo time shift (or thermal strain) increases with temperatures between 37 and $50^{\circ} \mathrm{C}$ in tissues ${ }^{4,9,15}$, and it has been applied to the development of systems and algorithms for monitoring HIFU ${ }^{16-18}$. However, some factors (e.g., thermal expansion, different intensity levels, and variations in speed of sound for different tissue types) affect estimations of the echo time shift ${ }^{19}$. Thermal strain can also be mixed up with mechanical strain if mechanical deformations are present ${ }^{20}$. Although high-frame-rate imaging has been proposed to address the problem of in vivo tissue motion ${ }^{21}$, sufficient system resources are required to support imaging and computations.

Compared with the echo time shift, the CBE may be more viable for ultrasound localization of the HIFU focal spot. The CBE is caused primarily by thermal dependencies of the backscatter coefficients for scatterers; it varies almost monotonically with temperatures from 37 to $50^{\circ} \mathrm{C}^{11}$. Conventionally, a CBE image is constructed by pixel-to-pixel calculation of the ratio of the backscattered energy between each temperature and the reference temperature ${ }^{13}$. Conventional CBE imaging is sensitive to changes in the signal features of backscattering and speckle motion; however, tracking and compensation of speckle motion may be ignored if CBE imaging is used only for visualizing temperature changes without considering the estimation accuracy for $\mathrm{CBE}^{22}$. After that, $\mathrm{CBE}$ imaging was further applied to monitoring HIFU, demonstrating the potential of CBE in HIFU focal spot localization $^{21,23}$; whereas, CBE imaging is accompanied by tail-shaped artifacts occurring below the focal spot when monitoring HIFU, degrading the image contrast to some degree ${ }^{23}$. Thus, considering the presence and unavoidability of CBE artifacts, we must strive to enhance CBE imaging contrast to benefit the localization of the HIFU focal spot without sacrificing accuracy in representing temperature change.

Conventionally, algorithms for ultrasound CBE imaging are designed in the spatial domain ${ }^{24,25}$. In general, a major drawback for spatial-domain CBE imaging is that the CBE computation is easily affected by noises, particularly when the CBE is measured in decibels $(\mathrm{dB})$ to describe the intensity ratio on a logarithmic scale. During HIFU treatment, noise sources include power electrical facilities and acoustic interferences, which produce unfavorable noises for ultrasound CBE imaging. Alternatively, estimating the CBE through a spectral comparison in the frequency domain may be a feasible strategy. Through a frequency-domain transformation, a frequency selection can be performed for data analysis to provide improved noise tolerance and image contrast for CBE imaging during HIFU focal spot monitoring.

This study proposes an algorithmic scheme for frequency-domain CBE imaging to localize the HIFU focal spot. In vitro phantom experiments were conducted to validate the proposed method. Compared with conventional CBE maps, frequency-domain CBE imaging provided an improved contrast for HIFU monitoring; the CBE value also preserved a high correlation with temperature changes.

\section{Materials and methods}

Experimental setup. The experimental system setup is illustrated in Fig. 1 and included a self-assembled HIFU system, a clinical ultrasound system (Model T3000, Terason Inc., Northborough, MA, USA), and a thermocouple (TM-947SD, Lutron Inc., Taipei, Taiwan). The HIFU system comprised a function generator (AFG 3022B, Tektronix Inc., Beaverton, OR, USA), a gain-variable power amplifier (150A100B, Amplifier Research, Souderton, PA, USA), a power meter (Model 4421, Bird Inc., Solon, OH, USA), and a HIFU probe (Sonic Concepts Inc., Bothell, WA, USA). The central frequency and focal length of the HIFU probe were $2.12 \mathrm{MHz}$ (bandwidth: $1.96-2.28 \mathrm{MHz}$ ) and $5.5 \mathrm{~cm}$, respectively. To generate the HIFU beam, the function generator was used to output a $2.12-\mathrm{MHz}$ continuous sinusoidal wave, which was then amplified by the power amplifier for 


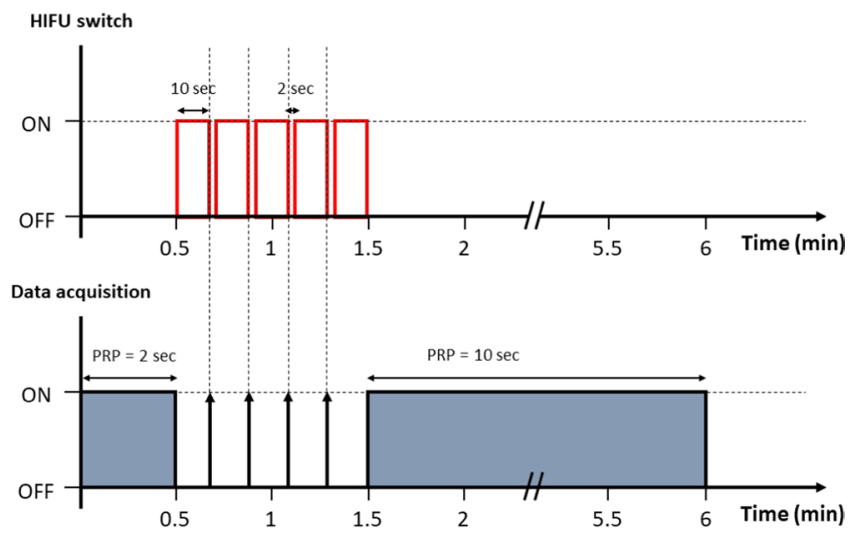

Figure 2. Sequence control for HIFU transmission and data acquisition, including the standby, heating, and cooling stages. To avoid the effect of the HIFU beam on ultrasound imaging, raw image data were acquired in each HIFU-off interval.

driving the HIFU probe. The driving power was monitored by the power meter. The clinical ultrasound system equipped with a convex array transducer (Model 5C2, Terason Inc., Northborough, MA, USA) was used for collecting ultrasound backscattered radiofrequency (RF) signals to monitor HIFU procedure. The imaging transducer frequency and the pulse length were $3 \mathrm{MHz}$ (bandwidth: $2.11-3.69 \mathrm{MHz}$ ) and $2.3 \mathrm{~mm}$, respectively. The thermocouple inserted into the phantom was responsible for measuring the temperature at the HIFU focal spot. The phantom was placed in an acrylic case, and a positioning hole was created on the wall of the case to facilitate the insertion of the thermocouple into the position where the imaging probe was confocal with the HIFU probe. The environmental temperature in the water tank, where the phantom was placed, was controlled by a temperature controller (I-629, ISTA Tech., Taoyuan, Taiwan) at approximately $33^{\circ} \mathrm{C}$.

Experimental procedures and data acquisition. Phantom experiments were performed to evaluate the performance of conventional spatial-domain CBE imaging and of the proposed frequency-domain CBE imaging in HIFU focal spot localization. The phantoms were prepared by adding $10 \mathrm{~g}$ of graphite powder (acoustic scatterers) with a diameter of $<20 \mu \mathrm{m}$ (Model 282863, Sigma-Aldrich, St. Louis, MO, USA) into agar solution produced by dissolving $2 \mathrm{~g}$ of agar powder in $200 \mathrm{~mL}$ of water. During the cooling period, a stirrer was used to suspend graphite powder in the agar solution until the solution was solidified. According to a previous study ${ }^{26}$, the applied ratio between the weight of graphite powder and the volume of the solution ensures the appearance of fully developed speckles in ultrasound B-mode images.

To simulate different thermal doses (temperature changes) at the HIFU focal spot, the driving voltages were set at 130,170 , and $210 \mathrm{mV}$ (peak-to-peak value), corresponding to the output power levels of 5, 10, and $15 \mathrm{~W}$, respectively. A phantom was placed in the water tank, and the thermocouple was inserted into the phantom for temperature monitoring. The imaging transducer was also immersed in the water and positioned above the phantom. Prior to the experiments, additional adjustments and calibrations were necessary for the experimental setup to allow both the thermocouple and the HIFU focal spot to be confocal and fall into the imaging plane. The experiments were initiated when the initial temperature of the phantom reached $33^{\circ} \mathrm{C}$. The sequence control for HIFU transmission and data acquisition can be divided into three stages, as presented in Fig. 2 . The first stage is defined as the standby period lasting $30 \mathrm{~s}$. In this stage, the HIFU system was off, and raw image data were acquired using the ultrasound imaging system every $2 \mathrm{~s}$. The first frame of the raw data was used as the reference for the analysis of CBE imaging. The HIFU system was turned on in the second stage, from the $31^{\text {st }}$ to the $90^{\text {th }} \mathrm{s}$, which comprised five 10-s heating phases with 2-s HIFU-off intervals between each heating phase. To avoid the effect of the HIFU beam on ultrasound imaging, the raw image data were acquired in each HIFU-off interval. In the third stage, from the $91^{\text {st }}$ to the $360^{\text {th }} \mathrm{s}$, the HIFU system was turned off for cooling the phantom; during this period, the raw image data were acquired every $10 \mathrm{~s}$. During HIFU procedure, the temperature data measured by the thermocouple were also collected.

The raw image data consisted of 128 A-lines of backscattered RF signals at a sampling rate of $12 \mathrm{MHz}$. The imaging depth and focal length were approximately 8 and $4 \mathrm{~cm}$, respectively. For each output power, a total of five independent phantoms were used to repeat measurements. The acquired ultrasound RF signals were further processed for B-mode and CBE imaging. The details of the algorithms are provided as below.

Ultrasound B-mode, spatial- and frequency-domain CBE imaging. For raw image data, the absolute value of the Hilbert transform for each backscattered RF signal was calculated to obtain the envelope image. An ultrasound B-mode image was formed using a log-compressed envelope image at a dynamic range of $60 \mathrm{~dB}$. In this study, the window-to-window computational scheme was selected because it supports the acquisition of local ultrasound signals with finite lengths for frequency-domain analysis. The algorithm is illustrated in Fig. 3, and the details are provided as follows ${ }^{24}$.

First, the envelope image $R_{k}$ at each time point $\left(k=0,1,2, \ldots, n ; R_{0}\right.$ represents the reference data) can be expressed as 


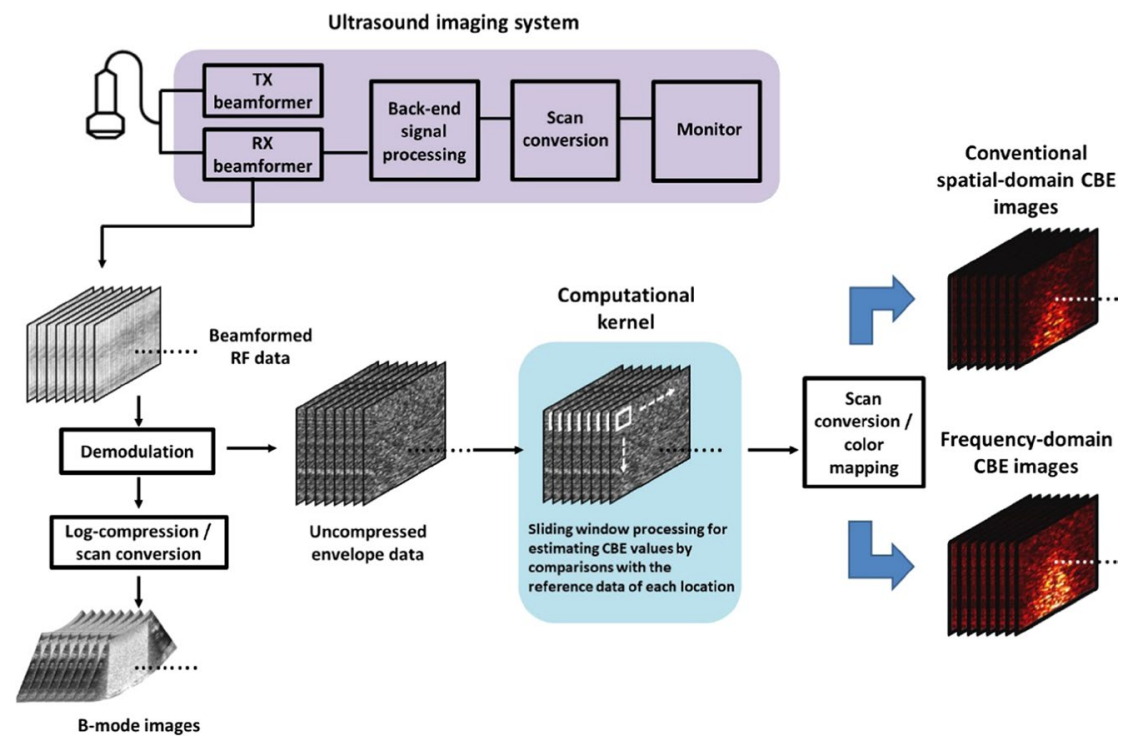

Figure 3. Algorithmic scheme for ultrasound B-mode, spatial-, and frequency-domain CBE imaging. The window-to-window computational scheme was used because it supports the acquisition of local ultrasound signals with finite lengths for frequency-domain analysis.

$$
R_{k}(x, y)=\left[\begin{array}{ccccc}
r_{k(1,1)} & r_{k(1,2)} & \cdot & \cdot & r_{k(1, x)} \\
r_{k(2,1)} & \cdot & \cdot & \cdot & \cdot \\
\vdots & : & : & \cdot & : \\
r_{k(y, 1)} & \cdot & \cdot & \cdot & r_{k(x, y)}
\end{array}\right] .
$$

To acquire local envelopes $\hat{R}_{\mathrm{k}}$, a window (i.e., subimage) with a size of $l \times m$ is set within $R_{k}$ :

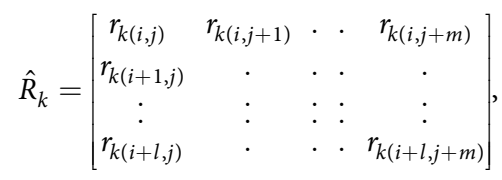

where $i$ and $j$ refer to locations in the upper left corner of the window. The local spatial-domain CBE value (denoted by $\hat{\eta}_{s}$ ) assigned as the new pixel located at the center of the window can be calculated using Eq. (3):

$$
\hat{\eta}_{s}=10 \log _{10}\left(\frac{E\left[\left(\hat{R}_{k}\right)^{2}\right]}{E\left[\left(\hat{R}_{0}\right)^{2}\right]}\right),
$$

where $E[\cdot]$ represents the statistical mean. The window then moves throughout $R_{k}$ and $R_{0}$ according to a window overlap ratio (WOR) for generating a CBE map. A spatial-domain CBE image with the same size as the original image can be obtained after data interpolation ${ }^{24}$ :

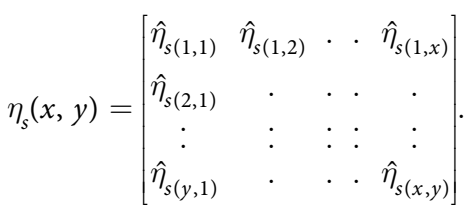

In this study, the WOR was set at 50\% to compromise CBE image resolution and computational complexity, and the side length corresponding to three times the pulse length of the ultrasound transducer $(6.9 \mathrm{~mm})$ was used to determine the dimension of the sliding window ${ }^{24}$.

For frequency-domain CBE imaging, the algorithmic scheme is nearly the same as that of the conventional approach in the spatial domain. The major difference is that the envelope image data serving as the input for the algorithm are replaced by the raw data of the backscattered RF signals for sliding window processing and Fourier transform computations. For each local RF signal acquired by the sliding window, the Fourier transform is performed and squared to obtain the power spectrum, which is used to establish the maximum magnitude in a gated frequency range between 2.5 and $3.5 \mathrm{MHz}$. Such a frequency range does not overlap with that of the HIFU probe, reducing the HIFU signal's effects on the $\mathrm{CBE}$ estimation. Thus, in the frequency domain, the local CBE value, denoted by $\hat{\eta}_{f}$, is estimated as follows: 


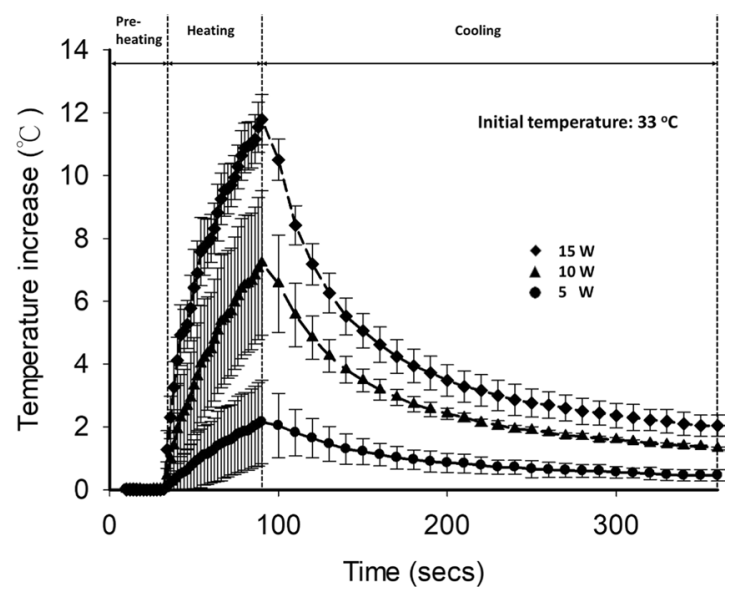

Figure 4. Temperature change measured close to the HIFU focal spot as a function of time for each output power. During HIFU heating, the temperatures increased by $2.2 \pm 1.2^{\circ} \mathrm{C}, 7.2 \pm 2.1^{\circ} \mathrm{C}$, and $11.8 \pm 0.7^{\circ} \mathrm{C}$ when the HIFU output power was 5,10 , and $15 \mathrm{~W}$, respectively.

$$
\hat{\eta}_{f}=10 \log _{10}\left(\frac{\max \left[\left|\mathrm{FT}\left(\hat{R}_{k}\right)\right|^{2}\right]}{\max \left[\left|\mathrm{FT}\left(\hat{R}_{0}\right)\right|^{2}\right]}\right),
$$

where $\max [\cdot]$ represents the maximum value in the predetermined bandwidth, and FT represents the Fourier transform. Using the sliding window technique for the spatial-domain CBE imaging, the frequency-domain CBE image can be obtained as

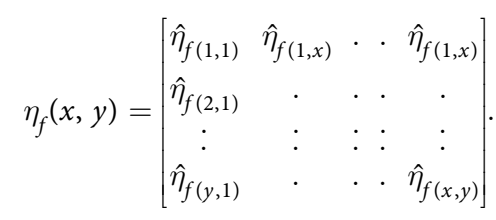

Data analysis. The temperature changes, which were measured using the thermocouple, as functions of time under HIFU exposures using different output powers were plotted. According to the previous study ${ }^{24}$, changes in the magnitude of the CBE during heating were evaluated by taking the absolute values of the spatial-and frequency-domain CBE images (denoted by $\left|\eta_{s}\right|$ and $\left|\eta_{f}\right|$, respectively). For each $\left|\eta_{s}\right|$ and $\left|\eta_{f}\right|$ image, a square region of interest (ROI) measuring $2 \mathrm{~mm} \times 2 \mathrm{~mm}$ was placed on the focal spot to calculate the average of the CBE magnitude (denoted by $\left|\eta_{s}\right|_{\text {focal spot }}$ and $\mid \eta_{f_{\text {focal spot }}}$, respectively) for temperature change comparisons. An ROI of the same size was also placed at a location $2 \mathrm{~cm}$ from the right side of the focal spot for calculating the CBE values of unheated regions (denoted by $\left|\eta_{s}\right|_{\text {background }}$ and $\left|\eta_{f}\right|_{\text {background }}$, respectively). A contrast factor was then calculated to evaluate the contrast of $\mathrm{CBE}$ imaging in monitoring the focal spot, as defined by

$$
\mathrm{CF}=20 \log _{10}\left(\frac{\left.\overline{\mid \eta}\right|_{\text {focal spot }}}{\left.\overline{|\eta|}\right|_{\text {background }}}\right) .
$$

The changes in the contrast factor with time obtained from $\left|\eta_{s}\right|$ and $\left|\eta_{f}\right|$ images were plotted. The Pearson correlation coefficient $r$ was calculated to evaluate the correlation between CBE values and temperature. An independent $t$-test was used to calculate the probability value $p$ identifying significant differences in contrast between spatial- and frequency-domain CBE images ( $p<0.05$ was considered statistically significant).

\section{Results}

Figure 4 demonstrates the temperature change measured close to the HIFU focal spot as a function of time for each output power. During the period of HIFU heating, the temperature increased by $2.2 \pm 1.2^{\circ} \mathrm{C}, 7.2 \pm 2.1^{\circ} \mathrm{C}$, and $11.8 \pm 0.7^{\circ} \mathrm{C}$ when exposed to HIFU of 5,10 , and $15 \mathrm{~W}$, respectively. The temperature started to decrease after HIFU heating was turned off and gradually returned to the initial temperature. Considering that the temperature variation during HIFU exposure is relatively limited, the thermocouple artifacts related to viscous heating could be ignored (also not observed in the images in Fig. 5).

Figure 5 depicts the typical ultrasound B-mode and CBE $\left(\left|\eta_{s}\right|\right.$ and $\left.\left|\eta_{f}\right|\right)$ images obtained at various stages of HIFU exposure. No CBE information was detected in the images prior to HIFU heating, as shown in the images at the $26^{\text {th }} \mathrm{s}$. The images at the $34^{\text {th }} \mathrm{s}$ referred to those obtained under the exposure of the HIFU beam and exhibited significant acoustic interference artifacts. During HIFU heating, the brightness of the $\left|\eta_{s}\right|$ and $\left|\eta_{f}\right|$ 

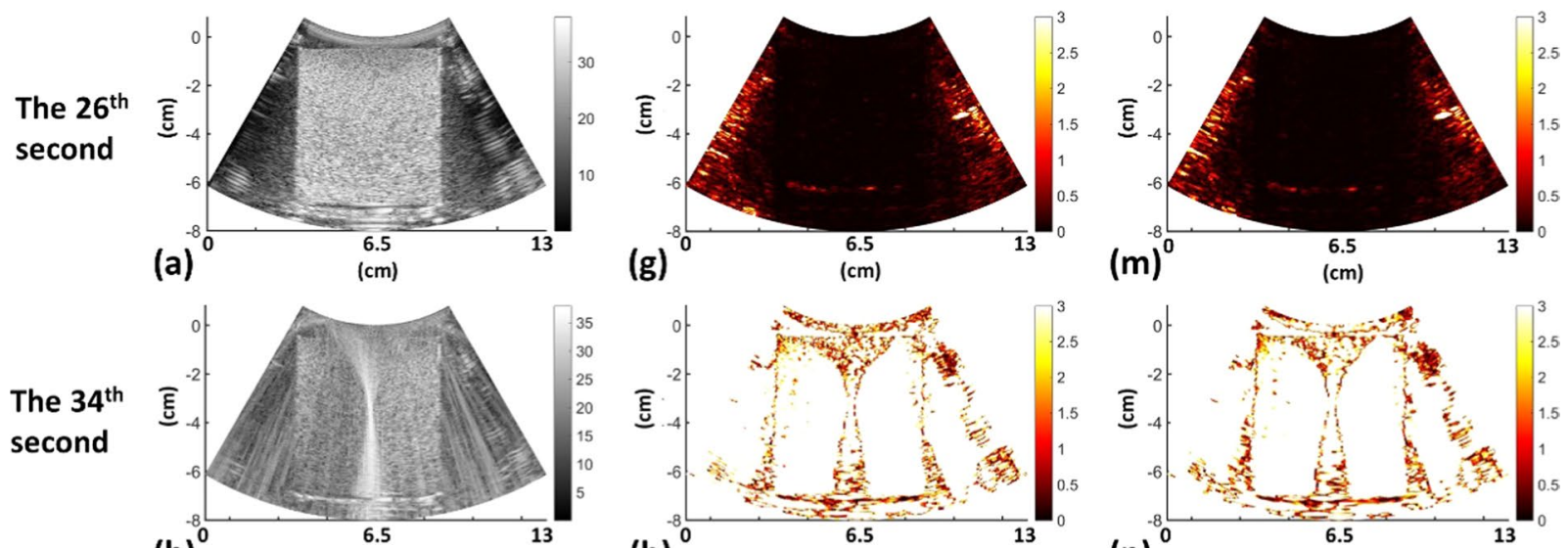

(b)

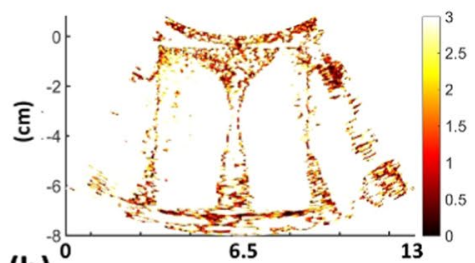

(h)
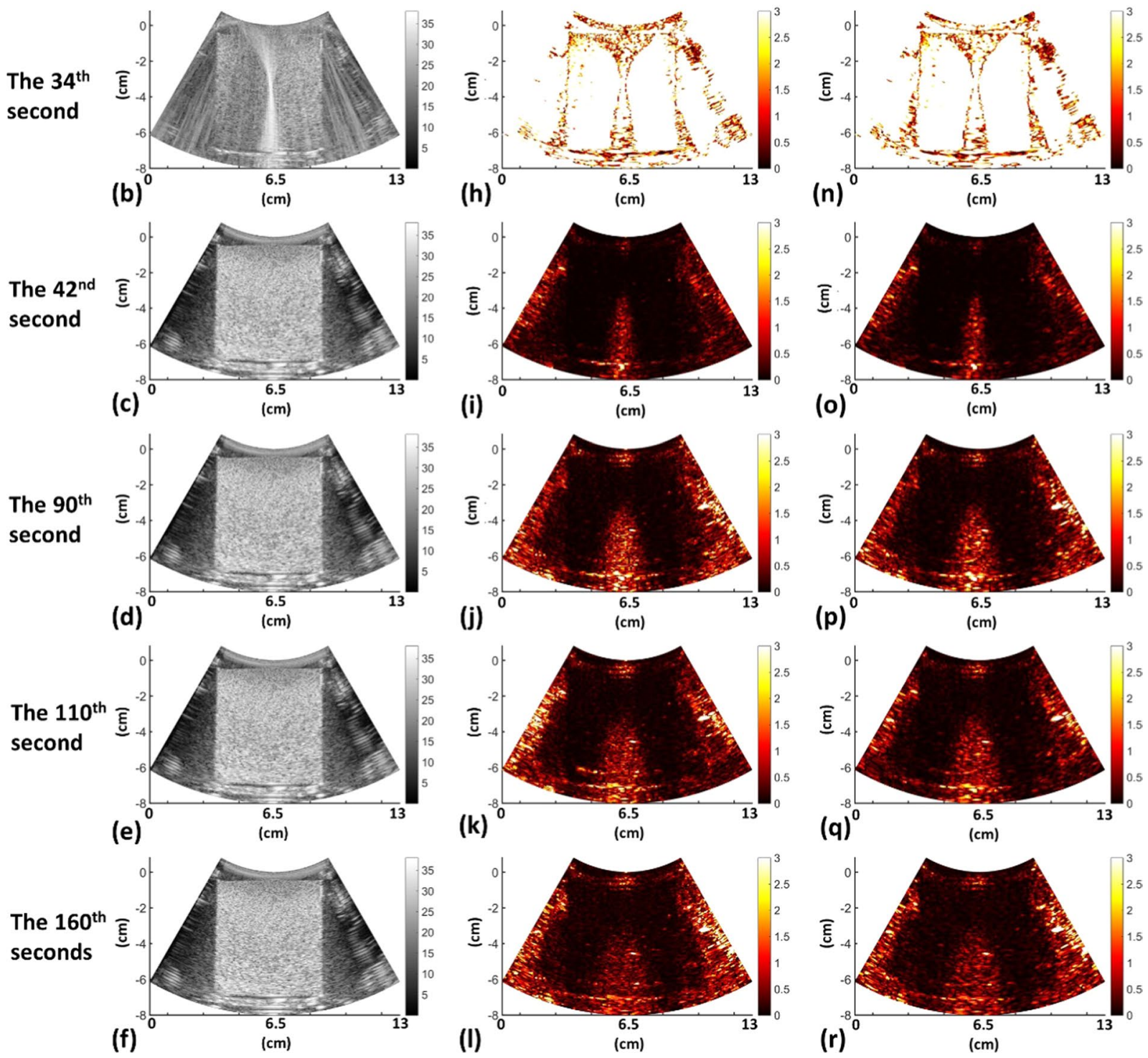

Figure 5. Typical ultrasound B-mode, spatial-, and frequency-domain CBE images obtained at different time points when 15-W HIFU was used. (a-f) B-mode; (g-l) spatial-domain; and (m-r) frequency-domain CBE images. Frequency-domain CBE imaging inherited the ability of the conventional spatial-domain approach in ultrasound thermal imaging.

images corresponding to the focal spot increased, signifying that spatial- and frequency-domain CBE images can visualize temperature changes, as shown in the images at the $42^{\text {nd }}$ and $90^{\text {th }} \mathrm{s}$ (the image data were collected in the HIFU-off intervals). However, tail-shaped artifacts were observed behind the HIFU focal spot with the two types of CBE imaging approaches. When HIFU heating was off, the overall brightness of the CBE images decreased, indicating that $\left|\eta_{s}\right|$ and $\left|\eta_{f}\right|$ images can also depict a decrease in temperature during the cooling period, as shown in the images at the $110^{\text {th }}$ and $160^{\text {th }}$ s.

Moreover, further analysis indicated that the frequency- and spatial-domain CBE values were closely correlated $(r=0.97)$, as shown in Fig. 6(a). For each power, the increase in temperature did not result in a significant monotonous change in the difference between $\left.\overline{\mid \eta_{s}}\right|_{\text {focal spot }}$ and $\left.\eta_{f}\right|_{\text {focal spot }}$ (Fig. 6(b)); in particular, the $\left|\eta_{f}\right|_{\text {focal spot }}$ value demonstrated a similar performance to the ${\overline{\mid \eta_{s}}}_{\text {focal spot }}$ value in describing temperature changes $(r=0.89)$, 

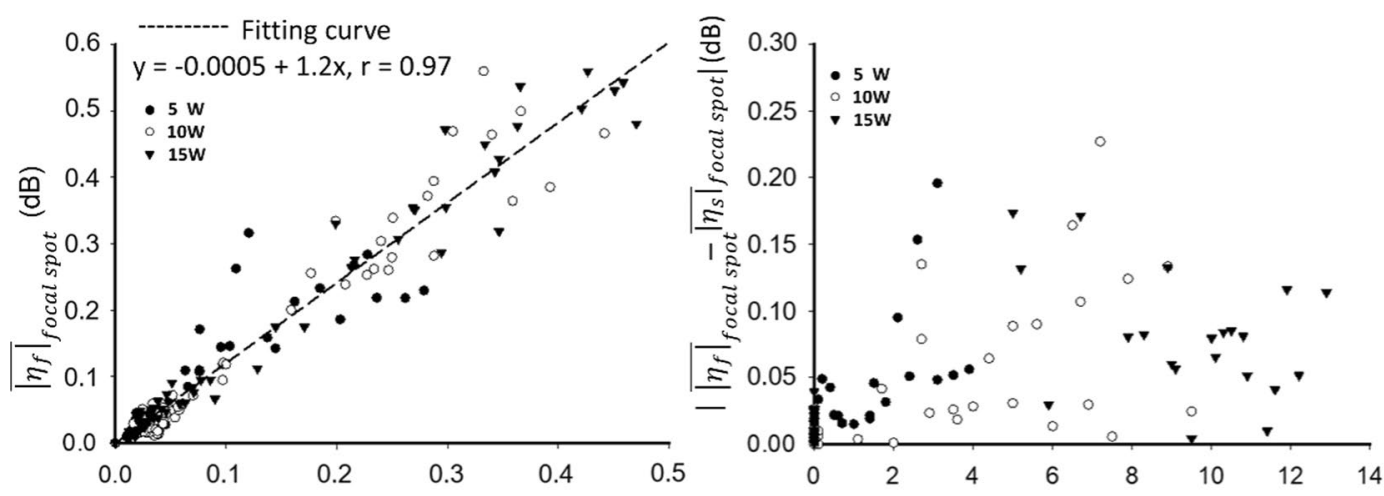

(a)

${\overline{\left|\eta_{s}\right|}}_{\text {focal spot }}(\mathrm{dB})$

(b)
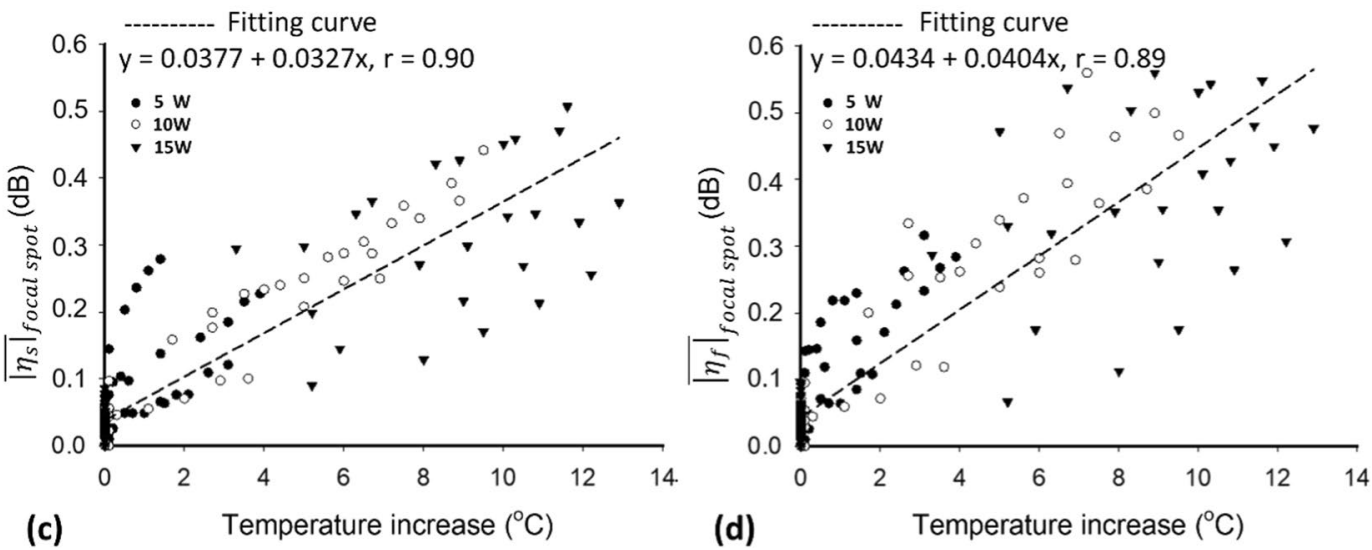

Figure 6. A total of the experimental data obtained from 5,10 , and $15 \mathrm{~W}$ were used for comparison analysis. (a) Correlation between $\left|\eta_{s}\right|_{\text {focal spot }}$ and $\mid \eta_{f_{\text {focal spot }}}$ values. The frequency- and spatial-domain CBE values were strongly correlated $(r=0.97)$. (b) Difference between the $\left|\eta_{s}\right|_{\text {focal spot }}$ and $\left|\eta_{f}\right|_{\text {focal spot }}$ values increased as the temperature change increased. (c) Relationship between the $\frac{\eta_{s} \mid}{\text { focal spot }}$ value and temperature change $(r=0.90)$. (d) Relationship between the $\mid \eta_{f_{\text {focal spot }}}$ value and temperature change $(r=0.89)$.

as supported by the results in Fig. 6(c,d). The aforementioned findings reveal that frequency-domain CBE imaging inherits the ability of the conventional spatial-domain approach in ultrasound thermal imaging.

To compare the performance of spatial- and frequency-domain CBE imaging in HIFU monitoring, the contrast factors of the $\left|\eta_{s}\right|$ and $\left|\eta_{f}\right|$ images as functions of time obtained at various output powers are depicted in Fig. 7. The $\left|\eta_{f}\right|$ image exhibited higher contrast factor values during the HIFU heating period than the conventional $\left|\eta_{s}\right|$ image did, signifying that frequency-domain CBE imaging yielded improved performance in visual localization of the HIFU focal spot. In particular, the group data comparison of the contrast factor between $\left|\eta_{s}\right|$ and $\left|\eta_{f}\right|$ imaging indicated that the image contrast obtained using the frequency-domain technique increased by 2.5 -fold $(4 \mathrm{~dB})$ compared with that obtained using conventional CBE imaging $(p<0.05)$, as shown in Fig. 8.

\section{Discussion}

Significance of this study. This study is the first to go beyond the spatial domain and develop an ultrasound CBE imaging method from the perspective of the frequency domain for reducing the effects of noises on conventional CBE imaging during HIFU treatment. The feasibility of the proposed method in HIFU focal spot localization was demonstrated through phantom experiments. The results showed that, compared with conventional spatial-domain CBE imaging, frequency-domain CBE imaging provided improved image contrast for HIFU monitoring; the CBE value also maintained a proportional relationship with temperature change.

Mechanisms of the CBE relationship with temperature. The physical mechanisms underlying the relationship between the CBE and temperature can be explained using two models proposed previously. According to the first model, the scatterers in biological soft tissues can be acoustically divided into lipid- and aqueous-based microstructures. With increasing temperature, lipid and aqueous scatterers contribute increasing and decreasing CBE values, respectively ${ }^{11-13}$. As mentioned in the Introduction, this is due to the thermal effects exerted on the backscatter coefficients of scatterers. The second model was proposed to describe the mechanism for the temperature response of the $\mathrm{CBE}^{27}$. The increase in temperature during heating alters the speed of sound, affecting the formation of backscattered waveforms resulted from wave interference. Under this condition, local changes in speckle patterns cause changes in CBE. Based on the current experimental design, the behaviors of the 
Spatial-domain CBE imaging

$5 W$

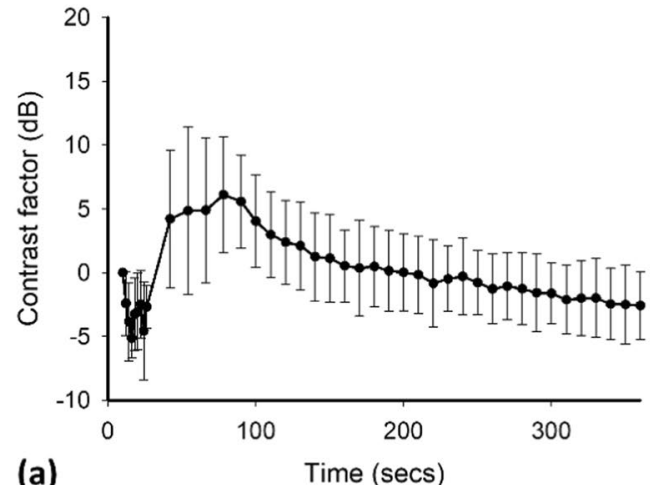

(a)

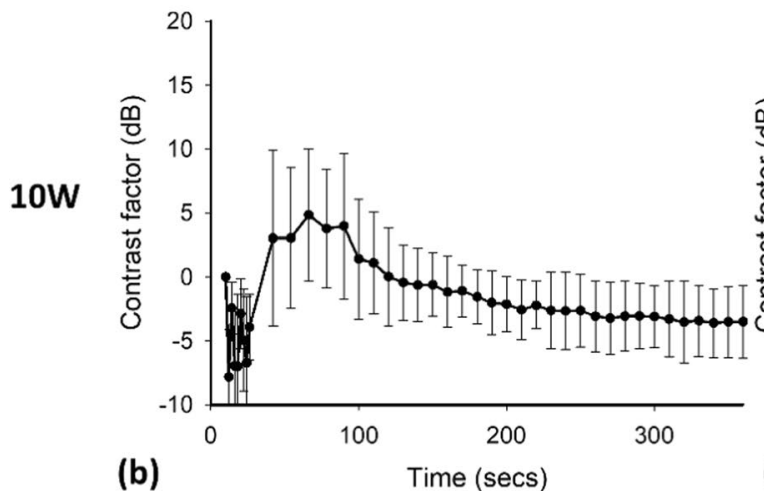

(b)

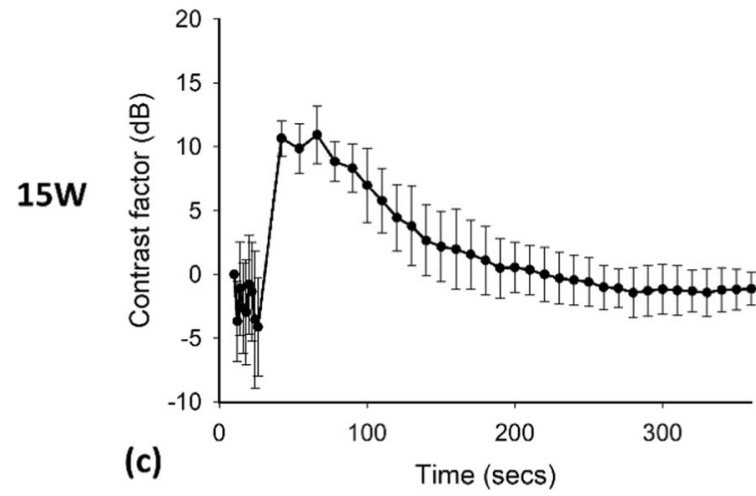

Frequency-domain CBE imaging

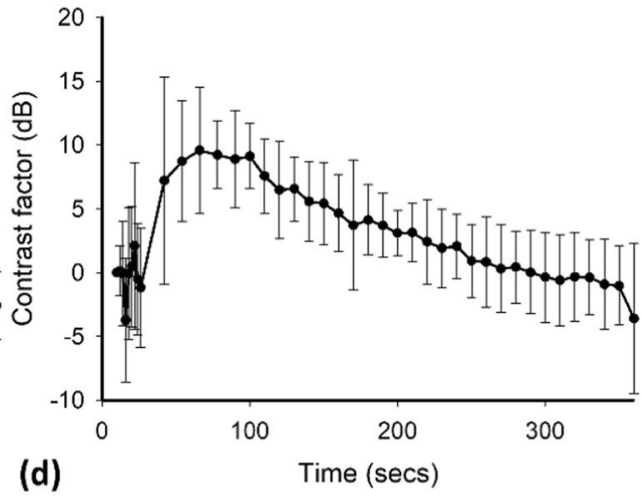

(d)
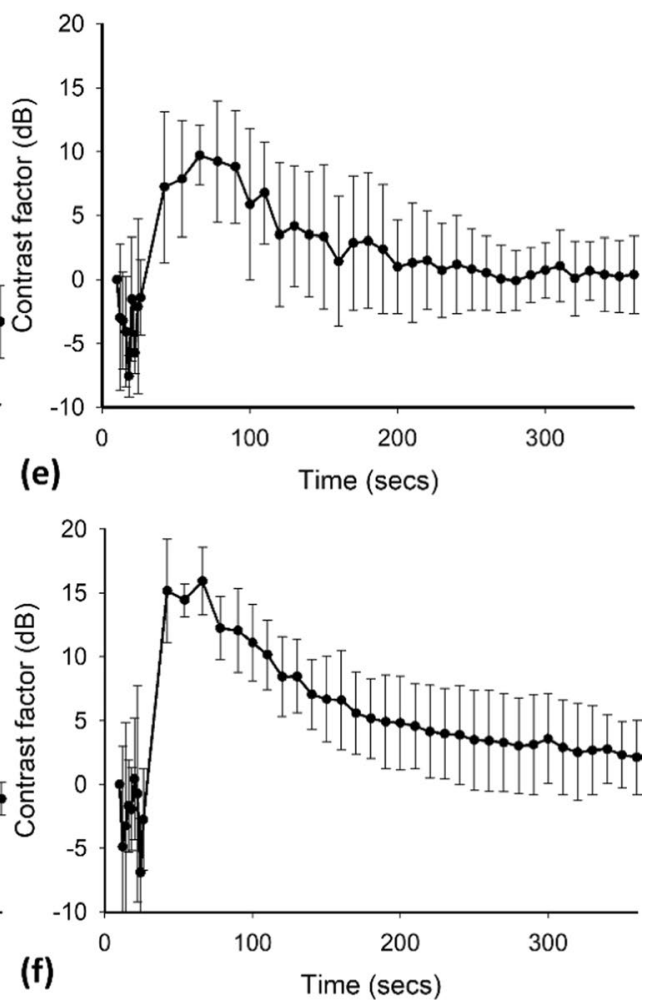

Figure 7. Contrast factors as functions of time obtained at different HIFU powers for $(\mathbf{a}-\mathbf{c})$ spatial-domain and (d-f) frequency-domain CBE imaging. The $\left|\eta_{f}\right|$ image exhibited higher contrast factor values in the period of HIFU heating than the conventional $\left|\eta_{s}\right|$ image did.

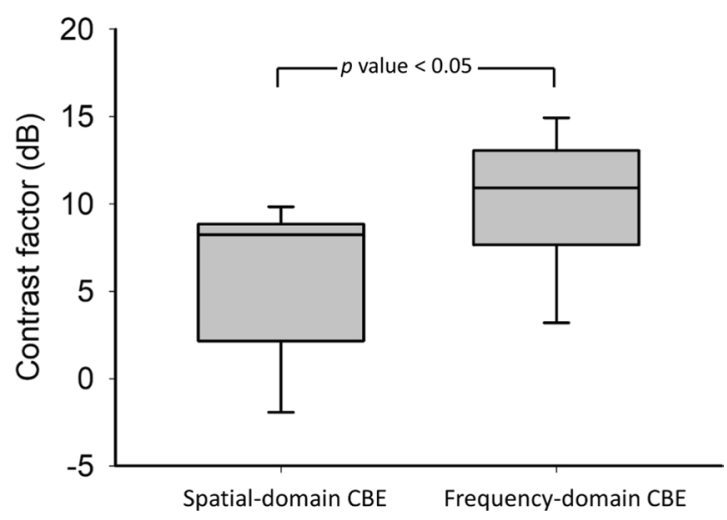

Figure 8. Box plots of contrast factors corresponding to spatial- and frequency-domain CBE images. Group data comparison of the contrast factor between $\left|\eta_{s}\right|$ and $\left|\eta_{f}\right|$ imaging indicated $p<0.05$. 
CBE during HIFU procedure can be explained by using the above two models that were established at temperatures between 37 and $50^{\circ} \mathrm{C}$. Currently, a more general CBE model that can support interpretations of the CBE for a wider temperature range is still not available. However, the effects of gas bubble formation, tissue necrosis, and stiffness increase will be dominant factors that need to be considered for high-temperature HIFU exposures.

Tail artifact in CBE imaging. In ultrasound thermal detection, reduced quality of ultrasound temperature imaging due to artifacts observed behind heated regions is an unavoidable problem. For example, when the echo time shift is used for imaging temperature change, ripple patterns typically appear under the heated region and severely corrupt temperature estimates at deeper locations ${ }^{9}$. In brief, the thermo-acoustic lens effect due to local changes in the sound speed (caused by temperature changes), like phase aberrations for the imaging system, results in a ripple. Therefore, smoothing along both axial and lateral directions has been recommended for the reduction of ripple artifacts ${ }^{9}$. Similar issues (tail-shaped artifacts) also affect ultrasound CBE imaging, regardless of whether spatial- or frequency-domain approaches are used, as per the current findings. However, tail artifacts in CBE images are not caused by the thermal lens effect; instead, they are caused by both refraction of ultrasound and changes in acoustic attenuation ${ }^{23}$. As suggested in a previous report ${ }^{23}$, tail artifacts can be partially suppressed by image fusion of both CBE and echo-time-shift mapping. Under this condition, replacing a conventional CBE approach with frequency-domain CBE imaging may further improve tail artifact suppression through the image fusion technique, because frequency-domain CBE imaging provides an improved contrast compared with conventional CBE imaging, as supported by the findings in this study.

Limitations and future work. Monitoring higher power HIFU treatment (such as HIFU ablation) was not considered; therefore, the use of frequency-domain CBE imaging for monitoring high-temperature changes is yet to be explored. HIFU heating ranging from 36 to $43^{\circ} \mathrm{C}$ was shown to activate drugs encapsulated by thermally sensitive liposomes ${ }^{21}$; therefore, the role of frequency-domain CBE imaging in HIFU-assisted drug delivery and release is worth exploring. Moreover, the initial temperature in each experiment was not controlled at a physiological temperature due to the limitation of the specification for the temperature controller. Such a condition may result in some unpredictable biases in estimating CBE values. However, this does not affect the primary conclusions on the advantages of frequency-domain CBE imaging. In addition, we believe that the suppression of artifacts in CBE imaging is a worthwhile and valuable development direction, but so far little research has been devoted to this topic, especially to solving the problem in the frequency domain. Finally, further investigation is merited using animal models to obtain additional validation. In particular, the effect of necrosis may occur and needs to be considered in practical applications to biological tissues. Tissue necrosis may result in acoustic attenuation, which further causes frequency downshift and changes in the amplitude distribution of the spectrum ${ }^{28}$. In this circumstance, the uncertainty may exist in the estimation of frequency-domain CBE if we include all the signal components in a specific frequency range for the analysis. Therefore, we used Eq. (5) as the definition of the frequency-domain CBE in this preliminary study. However, the algorithm can be flexibly modified according to future needs in practice (e.g., considerations of attenuation compensation).

\section{Conclusions}

This study proposed a new method for ultrasound CBE imaging based on frequency-domain analysis. Phantom experiments were conducted to validate the performance of the proposed frequency-domain CBE imaging in HIFU focal spot localization. The results showed that frequency-domain CBE imaging inherits the advantage of conventional spatial-domain CBE imaging in ultrasound thermal monitoring. However, the superiority of the frequency-domain approach over the conventional method was that the CBE map generated provided an improved contrast to visually monitor the HIFU focal spot. In the future, the technique of image construction in the frequency domain may be a new strategy for contrast enhancement in CBE imaging for focal spot localization in HIFU treatment planning. Tail-artifact-related problems should be resolved prior to subsequent practical applications.

Received: 17 April 2019; Accepted: 10 March 2020;

Published online: 25 March 2020

\section{References}

1. Wu, F. et al. Extracorporeal high intensity focused ultrasound ablation in the treatment of patients with large hepatocellular carcinoma. Ann. Surg. Oncol. 11, 1061-1069 (2004).

2. Illing, R. O. et al. The safety and feasibility of extracorporeal high-intensity focused ultrasound (HIFU) for the treatment of liver and kidney tumours in a Western population. Br. J. Cancer 93, 890-895 (2005).

3. O'Donnell, M. \& Flax, S. W. Phase aberration measurements in medical ultrasound: human studies. Ultrason. Imaging 10, 1-11 (1988).

4. Han, Y., Hou, G. Y., Wang, S. \& Konofagou, E. High intensity focused ultrasound (HIFU) focal spot localization using harmonic motion imaging (HMI). Phys. Med. Biol. 60, 5911-5924 (2015).

5. Bohris, C. et al. Quantitative MR temperature monitoring of high-intensity focused ultrasound therapy. Magn. Reson. Imaging 17, 603-610 (1999)

6. Chung, A. H. et al. Optimization of spoiled gradient-echo phase imaging for in vivo localization of a focused ultrasound beam. Magn. Reson. Med. 36, 745-752 (1996).

7. Maass-Moreno, R. \& Damianou, C. A. Noninvasive temperature estimation in tissue via ultrasound echo-shifts. Part I. Analytical model. J. Acoust. Soc. Am. 100, 2514-2521 (1996).

8. Maass-Moreno, R., Damianou, C. A. \& Sanghvi, N. T. Noninvasive temperature estimation in tissue via ultrasound echo-shifts. Part II. In vitro study. J. Acoust. Soc. Am. 100, 2522-2530 (1996).

9. Simon, C., Vanbaren, P. \& Ebbini, E. S. Two-dimensional temperature estimation using diagnostic ultrasound. IEEE Trans. Ultrason. Ferroelectr. Freq. Control. 45, 1088-1099 (1998). 
10. Tyréus, P. D. \& Diederich, C. Two-dimensional acoustic attenuation mapping of high-temperature interstitial ultrasound lesions. Phys. Med. Biol. 49, 533-546 (2004).

11. Straube, W. L. \& Arthur, R. M. Theoretical estimation of the temperature dependence of backscattered ultrasonic power for noninvasive thermometry. Ultrasound Med. Biol. 20, 915-922 (1994).

12. Arthur, R. M., Straube, W. L., Trobaugh, J. W. \& Moros, E. G. Non-invasive estimation of hyperthermia temperatures with ultrasound. Int. J. Hyperth. 21, 589-600 (2005).

13. Trobaugh, J. W., Arthur, R. M., Straube, W. L. \& Moros, E. G. A simulation model for ultrasonic temperature imaging using change in backscattered energy. Ultrasound Med. Biol. 34, 289-298 (2008).

14. Kaye, E. A., Chen, J. \& Pauly, K. B. Rapid MR-ARFI method for focal spot localization during focused ultrasound therapy. Magn. Reson. Med. 65, 738-743 (2011).

15. Seip, R. \& Ebbini, E. S. Noninvasive estimation of tissue temperature response to heating fields using diagnostic ultrasound. IEEE Trans. Biomed. Eng. 42, 828-839 (1995).

16. Anand, A., Savéry, D. \& Hall, C. Three-dimensional spatial and temporal temperature imaging in gel phantoms using backscattered ultrasound. IEEE Trans. Ultrason. Ferroelectr. Freq. Control. 54, 23-31 (2007).

17. Ding, X. et al. Improved estimation of ultrasound thermal strain using pulse inversion harmonic imaging. Ultrasound Med. Biol. 42, $1182-1192(2016)$.

18. Foiret, J. \& Ferrara, K. W. Spatial and temporal control of hyperthermia using real time ultrasonic thermal strain imaging with motion compensation, phantom study. PLoS ONE 10, e0134938 (2015).

19. Rangraz, P., Behnam, H., Sobhebidari, P. \& Tavakkoli, J. Real-time monitoring of high-intensity focused ultrasound thermal therapy using the manifold learning method. Ultrasound Med. Biol. 40, 2841-2850 (2014).

20. Seo, C. H., Shi, Y., Huang, S. W., Kim, K. \& O’Donnell, M. Thermal strain imaging: a review. Interface Focus. 1, 649-664 (2011).

21. Seo, J. et al. Motion compensation for ultrasound thermal imaging using motion-mapped reference model: an in vivo mouse study. IEEE Trans. Biomed. Eng. 61, 2669-2678 (2014).

22. Tsui, P. H., Chien, Y. T., Liu, H. L., Shu, Y. C. \& Chen, W. S. Using ultrasound CBE imaging without echo shift compensation for temperature estimation. Ultrasonics 52, 925-935 (2012).

23. Choi, K., Kong, D., Park, J., Cho, J. \& Lee, H. K. Noninvasive ultrasound temperature imaging with fusion algorithm. IEEE Ultrasonics Symposium, 933-936 (2012).

24. Zhang, L., Li, Q., Wang, C. Y. \& Tsui, P. H. Ultrasound single-phase CBE imaging for monitoring radiofrequency ablation. Int. J. Hyperth. 35, 548-558 (2018).

25. Xia, J., Li, Q., Liu, H. L., Chen, W. S. \& Tsui, P. H. An approach for the visualization of temperature distribution in tissues according to changes in ultrasonic backscattered energy. Comput. Math. Methods Med. 2013, 682827 (2013).

26. Tsui, P. H. et al. Small-window parametric imaging based on information entropy for ultrasound tissue characterization. Sci. Rep. 7 , 41004 (2017)

27. Li, X., Ghoshal, G., Lavarello, R. J. \& Oelze, M. L. Exploring potential mechanisms responsible for observed changes of ultrasonic backscattered energy with temperature variations. Med. Phys. 41, 052901 (2014).

28. Shung, K. K. Diagnostic ultrasound: imaging and blood flow measurements. CRC Press, Taylor \& Francis Group, Abingdon (2015).

\section{Acknowledgements}

This work was supported by the Ministry of Science and Technology in Taiwan (Grant Nos. MOST 106-2221-E-182-023-MY3, MOST 108-2218-E-182-001, and MOST 107-2221-E-182A-001) and the Chang Gung Memorial Hospital at Linkou in Taiwan (Grant Nos. CMRPD1H0381 and CMRPG3F2131). This work was also supported in part by the National Natural Science Foundation of China (Grant Nos. 11804013, 61871005, and 61471263), the Beijing Natural Science Foundation (Grant No. 4184081), and the Tianjin Natural Science Foundation (Grant No. 16JCZDJC31100).

Author contributions

K.Y., Z.Z., Q.L. and C.K.C. wrote the manuscript text and prepared figures. H.L.L. and P.H.T. supported the HIFU facilities and designed the measurement protocol. C.W.H., J.R.C. and Y.W.T. worked on the experiments and data analysis. All authors reviewed the manuscript.

\section{Competing interests}

The authors declare no competing interests.

\section{Additional information}

Correspondence and requests for materials should be addressed to Z.Z. or P.-H.T.

Reprints and permissions information is available at www.nature.com/reprints.

Publisher's note Springer Nature remains neutral with regard to jurisdictional claims in published maps and institutional affiliations.

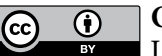

Open Access This article is licensed under a Creative Commons Attribution 4.0 International

License, which permits use, sharing, adaptation, distribution and reproduction in any medium or format, as long as you give appropriate credit to the original author(s) and the source, provide a link to the Creative Commons license, and indicate if changes were made. The images or other third party material in this article are included in the article's Creative Commons license, unless indicated otherwise in a credit line to the material. If material is not included in the article's Creative Commons license and your intended use is not permitted by statutory regulation or exceeds the permitted use, you will need to obtain permission directly from the copyright holder. To view a copy of this license, visit http://creativecommons.org/licenses/by/4.0/.

(C) The Author(s) 2020 